\title{
Quantum phases of spin-1 system on 3/4 and 3/5 skewed ladders
}

Cite as: J. Appl. Phys. 129, 223902 (2021); https://doi.org/10.1063/5.0048811

Submitted: 26 February 2021. Accepted: 19 May 2021 . Published Online: 08 June 2021

Sambunath Das, (D) Dayasindhu Dey, S. Ramasesha, and (iD) Manoranjan Kumar

\section{COLLECTIONS}

Paper published as part of the special topic on Spin Transition Materials: Molecular and Solid-State
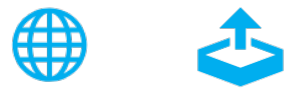

\section{ARTICLES YOU MAY BE INTERESTED IN}

The optimized memristor performance by utilizing the constrained domain wall motion in Pt/ $\mathrm{Co}-\mathrm{Tb} / \mathrm{Ta}$ structure

Journal of Applied Physics 129, 223901 (2021); https://doi.org/10.1063/5.0049928

On the temperature-dependent characteristics of perpendicular shape anisotropy-spin transfer torque-magnetic random access memories

Journal of Applied Physics 129, 223903 (2021); https://doi.org/10.1063/5.0054356

Negative ion sources

Journal of Applied Physics 129, 221101 (2021); https://doi.org/10.1063/5.0049289

Challenge us.

What are your needs for periodic signal detection?

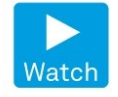

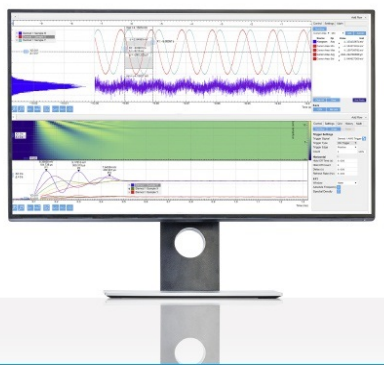

Zurich

- Instruments 


\title{
Quantum phases of spin-1 system on 3/4 and 3/5 skewed ladders
}

Cite as: J. Appl. Phys. 129, 223902 (2021); doi: 10.1063/5.0048811

Submitted: 26 February 2021 . Accepted: 19 May 2021 .

Published Online: 8 June 2021

Sambunath Das, ${ }^{1, a)}$ Dayasindhu Dey, ,b) (D S. Ramasesha, ${ }^{1, c)}$ and Manoranjan Kumar ${ }^{2, d)}$ (D)

\section{AFFILIATIONS}

${ }^{1}$ Solid State and Structural Chemistry Unit, Indian Institute of Science, Bangalore 560012, India

${ }^{2}$ S. N. Bose National Centre for Basic Sciences, Block-JD, Sector-III, Salt Lake, Kolkata 700106, India

Note: This paper is part of the Special Topic on Spin Transition Materials: Molecular and Solid-State.

a) sambunath.das46@gmail.com

b)dayasindhu.dey@gmail.com

c)ramasesh@iisc.ac.in

${ }^{d)}$ Author to whom correspondence should be addressed: manoranjan.kumar@bose.res.in

\begin{abstract}
We study the quantum phase transitions of frustrated antiferromagnetic Heisenberg spin-1 systems on the $3 / 4$ and $3 / 5$ skewed two leg ladder geometries. These systems can be viewed as arising by periodically removing rung bonds from a zigzag ladder. We find that in large systems, the ground state (gs) of the $3 / 4$ ladder switches from a singlet to a magnetic state for $J_{1} \geq 1.82$; the gs spin corresponds to the ferromagnetic alignment of effective $S=2$ objects on each unit cell. The gs of antiferromagnetic exchange Heisenberg spin-1 system on a $3 / 5$ skewed ladder is highly frustrated and has spiral spin arrangements. The amplitude of the spin density wave in the $3 / 5$ ladder is significantly larger compared to that in the magnetic state of the 3/4 ladder. The gs of the system switches between singlet state and low spin magnetic states multiple times on tuning $J_{1}$ in a finite size system. The switching pattern is nonmonotonic as a function of $J_{1}$ and depends on the system size. It appears to be the consequence of a higher $J_{1}$ favoring a higher spin magnetic state and the finite system favoring a standing spin wave. For some specific parameter values, the magnetic gs in the $3 / 5$ system is doubly degenerate in two different mirror symmetry subspaces. This degeneracy leads to spontaneous spin-parity and mirror symmetry breaking, giving rise to spin current in the gs of the system.
\end{abstract}

Published under an exclusive license by AIP Publishing. https://doi.org/10.1063/5.0048811

\section{INTRODUCTION}

Spin chains and ladders show strong quantum fluctuations due to spatial confinement and are extensively studied to explore the intriguing magnetic properties of these systems. The excitations and ground state (gs) properties of these systems depend on the magnitude of the spins at the lattice sites (half-odd-integer or integer $^{1-4}$ and topology of the exchange interactions. ${ }^{5-26} \mathrm{~A}$ Heisenberg antiferromagnetic (HAF) spin-1/2 chain with only nearest neighbor exchange interaction, $J_{1}$, shows a quasi-long range order in the gs and a gapless spectrum, ${ }^{27}$ whereas a spin-1/2 HAF chain with nearest neighbor and next nearest neighbor exchange interactions $J_{1}$ and $J_{2}$, respectively, can show quasi-long range or short range order and gapless or gapped spectrum depending on the ratio $J_{2} / J_{1} \cdot{ }^{5-9}$ The HAF spin-1 chains, on the other hand, with only nearest neighbour exchange interaction have short range order in the gs and gapped spectrum as pointed out by Haldane, ${ }^{1,2}$ and gs can be represented as a valance bond solid (VBS), ${ }^{3,4,28}$ which is of the same universality class as the Affleck, Kennedy, Lieb, and Tasaki (AKLT) states. ${ }^{3,4}$ The AKLT state has inspired many numerical techniques like matrix product states, ${ }^{29-31}$ tensor network methods, ${ }^{32}$ and projected entangled pair states (PEPS) methods. ${ }^{30,33}$ AKLT states can also be represented as cluster states, which can be used in measurement-based quantum computation. $^{34,35}$

The first experimental realization of the spin-1 Haldane system was in the well known transition metal chain compound $\mathrm{Ni}\left(\mathrm{C}_{2} \mathrm{H}_{8} \mathrm{~N}_{2}\right)_{2} \mathrm{NO}_{2} \mathrm{ClO}_{4}$ (NENP). ${ }^{36-38}$ The HAF spin-1 chain exhibits topological phase with spin-1/2 edge modes leading to fourfold degenerate gs in the thermodynamic limit. In spin-1 chain, the gs correlation length $\xi=6.05$ lattice constant and a large spin gap to the excited state $\Delta_{\mathrm{ST}} \sim 0.41 J_{1} \cdot{ }^{39,40}$ The gs of a two leg HAF spin-1 
normal ladder is a plaquette-singlet solid state (PSSS), where two spin-1/2 singlet dimers are sitting at each rung and there is no overlap to the VBS state in the large rung exchange limit. ${ }^{41}$ On a zigzag ladder, the gs of a spin-1 HAF model is the Haldane phase in the weak rung interaction limit, while a double Haldane state is the gs in the large rung interaction limit. ${ }^{42,43}$ Another class of spin ladders that have been studied in recent times is the skewed ladder, which contains slanted rung bonds that periodically displace rung bonds as well as periodically missing rung bonds of the original ladder (Fig. 1). This leads to fused equivalent or inequivalent cyclic rings. An example of such a skewed ladder is the 5/7 skewed ladder with alternately fused five- and seven-membered rings while the $5 / 5$ skewed system consists of equivalent five membered rings fused together. The $5 / 7$ system has been studied in both spin- $1 / 2^{44-46}$ and spin- 1 cases. ${ }^{47}$ Similarly, the $3 / 4,3 / 5$, and 5/5 spin-1/2 skewed ladders have also been studied earlier and it was shown that the gs of heteroskewed ladders (polygons of unequal number of vertices fused together), such as 5/7 and $3 / 4$, are magnetic. ${ }^{45}$ In the large rung exchange limit, the gs wavefunction of $3 / 4$ skewed ladder can be represented as a product of rung singlet dimers and one ferromagnetically interacting spin- $1 / 2$ object per triangle. ${ }^{45}$ The gs of a spin-1/2 system on a $3 / 5$ geometry is a low spin magnetic state for intermediate values of $J_{1}$ but evolves to a gapless antiferromagnetic state for large $J_{1} \cdot{ }^{45}$ As outlined above, the spin-1/2 and spin-1 systems exhibit fundamentally different behavior in the case of HAF spin chains. This has prompted us to study the spin-1 system on these ladders to explore the quantum phase transitions in these systems as the ratio of the rung exchange to the ladder exchange strengths is varied.

In this paper, we focus on the gs properties of spin-1 objects arranged on $3 / 4$ and $3 / 5$ skewed ladders interacting via competing

(a)

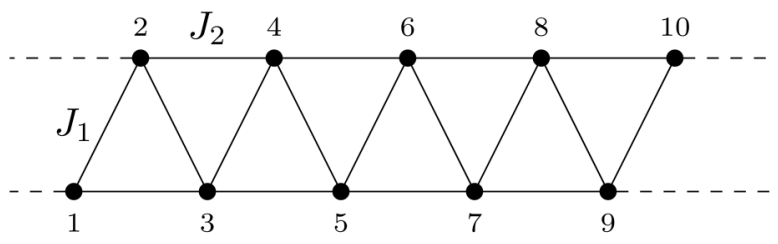

(b)

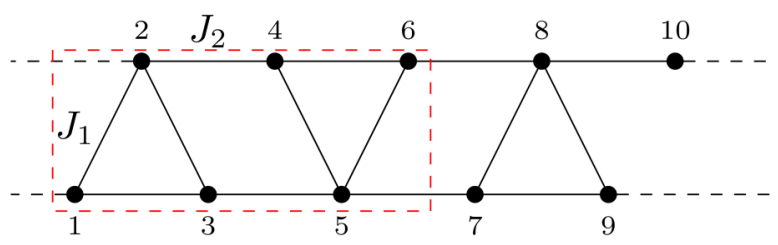

(c)

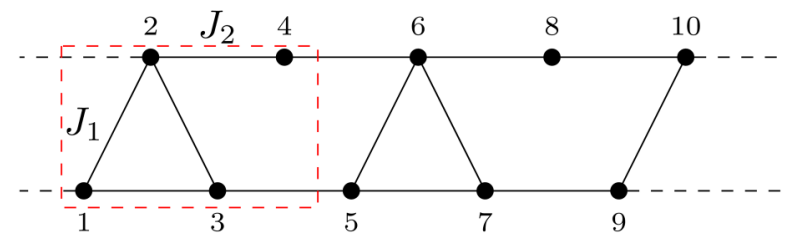

FIG. 1. Schematic diagram of (a) zigzag ladder, (b) 3/4 skewed ladder, and (c) 3/5 skewed ladder. The unit cell of each structure is shown inside the box in red. rung and leg antiferromagnetic exchange interactions of strength $J_{1}$ and $J_{2}$, respectively. The structures of zigzag, $3 / 4$, and $3 / 5$ skewed ladders are shown in Figs. 1(a)-1(c), respectively. In Sec. II, we discuss the model Hamiltonian and numerical methods used in the paper. The results for $3 / 4$ and $3 / 5$ skewed ladders are discussed in Sec. III. In Sec. III A, we show that the gs of the $3 / 4$ system switches from a singlet to a magnetic state for $J_{1} \geq 1.82$ with magnetization per unit cell " $\langle m\rangle$ ” taking a value of 2 . In Sec. III B, we show that the gs of 3/5 ladder exhibits short range spin correlations and a spiral spin density wave, and the spin of the gs depends both on the size of the system and $J_{1}$ for intermediate $J_{1}$ values; the gs is magnetic with one unpaired spin per unit cell in the large $J_{1}$ limit. For some specific $J_{1}$ values, the gs is degenerate and exhibits vector chiral phase due to simultaneous breaking of spin inversion and reflection symmetries. Section IV provides a summary of results and conclusions.

\section{MODEL AND METHOD}

We consider the HAF spin-1 model on $3 / 4$ and $3 / 5$ skewed ladders shown in Figs. 1(b) and 1(c), respectively; all exchange interactions between the neighboring spins are antiferromagnetic in nature. The site numberings are shown in Figs. 1(b) and 1(c). The exchange interactions along rung and leg bonds are represented as $J_{1}$ and $J_{2}$, respectively. The exchange interaction $J_{2}$ is set to 1 to define the energy scale in all the studies. The model Hamiltonian of $3 / 4$ skewed ladder can be written as

$$
H_{3 / 4}=J_{1} \sum_{k=1}^{N / 3} \vec{S}_{3 k-1} \cdot\left(\vec{S}_{3 k-2}+\vec{S}_{3 k}\right)+J_{2} \sum_{k=1}^{N-2} \vec{S}_{k} \cdot \vec{S}_{k+2}
$$

where the system consists of $N=6 n$ sites, with $n$ being the number of unit cells and an open boundary condition (OBC) is assumed [Fig. 1(b)]. The first term denotes the rung exchange and the second term denotes the exchange interactions along the legs. The model Hamiltonian of 3/5 skewed ladder can be written as

$$
H_{3 / 5}=J_{1} \sum_{i=1}^{n} \vec{S}_{4 i-2} \cdot\left(\vec{S}_{4 i-3}+\vec{S}_{4 i-1}\right)+J_{1} \vec{S}_{4 n+2} \cdot \vec{S}_{4 n}+J_{2} \sum_{i=1}^{4 n} \vec{S}_{i} \cdot \vec{S}_{i+2} \text {, }
$$

where $n$ is the number of unit cells and an open boundary condition is assumed. In the case of periodic boundary condition, the Hamiltonian is modified accordingly and sites $4 i-2$ and $4 i$ are the inversion centers of the system. Both $\mathrm{H}_{3 / 4}$ and $\mathrm{H}_{3 / 5}$ conserve the total spin $S^{2}$ and the $z$-component of the total spin $S^{z}$.

We use the exact diagonalization (ED) technique for solving these ladders with up to 16 spins with periodic boundary condition (PBC). For larger system sizes, we use the now well known finite density matrix renormalization group (DMRG) method, ${ }^{48-51}$ which retains the size of the Hamiltonian matrix at all system sizes. This is achieved by a systematic truncation of the irrelevant degrees of freedom by selecting " $m$ " states with largest eigenvalues of the density matrix to span the Fock space of a part of the full system. The chosen value of $m$ is up to 500 in our studies and it keeps the truncation error of the density below $\sim 10^{-10}$. We also carry out 
6-10 finite sweeps for convergence. For some values of $J_{1}$ and for large system sizes, we have employed $m=1600$ and 12 finite DMRG sweeps to improve convergence. The largest system size used in this paper is $N=98$ or 24 unit cells with open boundary condition.

\section{RESULTS AND DISCUSSIONS}

In this section, we present the results for both $3 / 4$ and $3 / 5$ skewed ladders. The gs of the $3 / 4$ skewed ladder shows a transition from a singlet to a magnetic phase, whereas the gs of 3/5 ladder has spiral spin arrangement and switches between different magnetic and singlet states upon tuning $J_{1}$. In the decoupled limit, the gs of both systems show Haldane phase, whereas in the large $J_{1}$ limit, the gs exhibits strong rung trimer formation. In strong rung coupling limit (very large $J_{1}$ ), the gs of the system has an effective spin-1 on each triangle with those triangles interacting ferromagnetically. To analyze the magnetic transitions, various quantities like gs energies $E_{g s}$, energy gaps to low-lying excited states $\Gamma_{l}$, bond orders $b_{i, j}$ between sites $i$ and $j$, and local spin densities $\rho_{i}$ at site $i$ are analyzed as a function of $J_{1}$. The excitation gaps $\Gamma_{l}$ are defined as

$$
\Gamma_{l}=E_{0}\left(S^{z}=l\right)-E_{0}\left(S^{z}=0\right),
$$

where $E_{0}\left(S^{z}=l\right)$ and $E_{0}\left(S^{z}=0\right)$ are the energies of the lowest states in the $S^{z}=l$ and $S^{z}=0$ manifold, respectively, and $l$ is an integer. The model Hamiltonians in Eqs. (1) and (2) are isotropic and, therefore, conserve total spin $S$ and its $z$-component $S^{z}$. For a magnetic gs with spin $S, E_{0}\left(S^{z}=S\right)$ is degenerate with $E_{0}\left(S^{z}=m\right)$ where $-S \leq m \leq S$ and the state is $(2 S+1)$ fold degenerate. Thus, $S$ is the gs spin if the lowest energy level in every $S^{z} \leq S$ are the same or $\Gamma_{l}=0$ for all $l \leq S$. The bond order $b_{i j}=-\left\langle\vec{S}_{i} \cdot \vec{S}_{j}\right\rangle$, the spin density $\rho_{i}=\left\langle S_{i}^{z}\right\rangle$, and the correlation function $C(r)=$ $\left\langle\vec{S}_{i} \cdot \vec{S}_{i+r}\right\rangle$ are the gs expectation values calculated to characterize the gs with $S^{z}=S$. Due to symmetry, there are only two unique sites and three unique bonds per unit cell in the $3 / 4$ ladder, whereas there are three unique sites and four bonds in the $3 / 5$ ladder. Therefore, it is sufficient to obtain all of the above quantities for these unique sites and bonds.

\section{A. 3/4 skewed ladder}

The magnetic gs of the $3 / 4$ skewed ladder is obtained using excitation gaps $\Gamma_{l}$, defined in Eq. (3). In this system, the gs rapidly evolves from a singlet state to a magnetic state with spin $S=2 n$, where $n$ is the number of unit cells in the system. This transition seems continuous in systems with open boundary condition (OBC) with system size $N=50$. In this system [Fig. 2(a)], the gs spin $S_{G}$ changes gradually beyond $J_{1}=1.67$, and near $J_{1} \sim 1.75, S_{G}$ increases rapidly and beyond $J_{1}=1.82, S_{G}$ achieves the highest gs spin of $S_{G}=16$. The transition region shrinks dramatically to $1.65<J_{1}<1.67$ for $N=18$ with periodic boundary condition $(\mathrm{PBC})$, and for both $\mathrm{PBC}$ and $\mathrm{OBC}$ cases, the transition region decreases with the system size. The scaling of the total spin $S_{G}$ of the gs is shown as a function of unit cell in two parameter regimes in Fig. 2(b). The gs remains a singlet for $J_{1}<1.6$, whereas for larger $J_{1} \geq 1.82, S_{G}$ vs $N$ shows a linear variation with slope $1 / 3$.
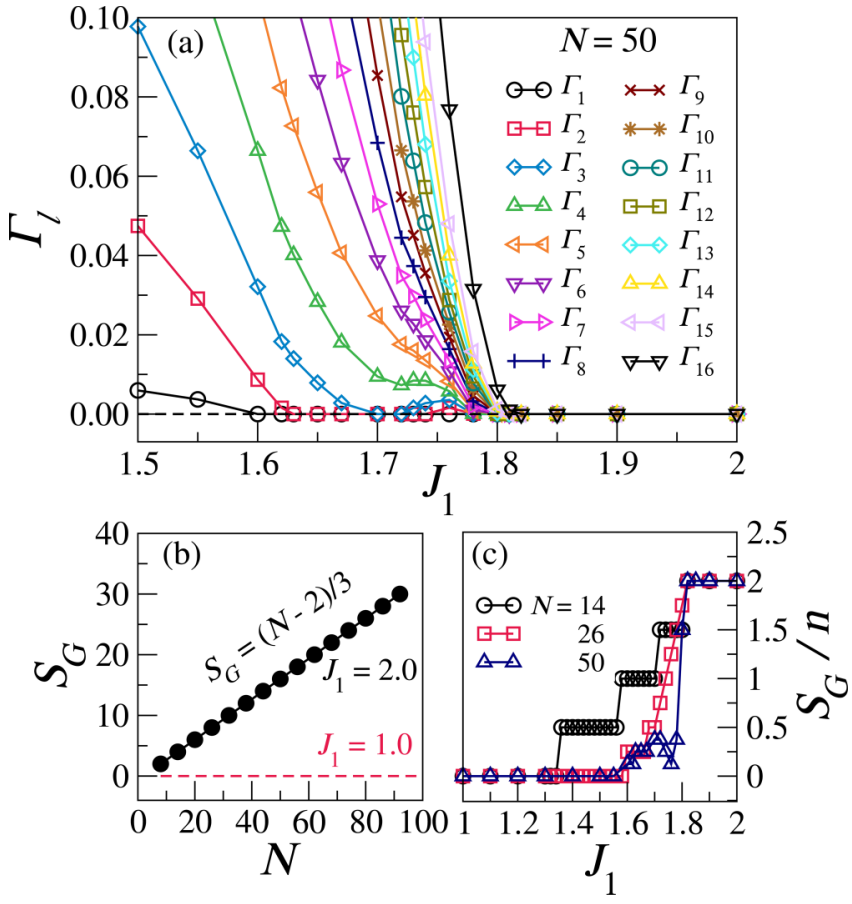

FIG. 2. (a) The lowest excitation gaps $\Gamma_{l}$ are shown as a function of $J_{1}$ for $3 / 4$ ladder of 50 sites with open boundary condition. For $J_{1}<1.6$, all the $\Gamma_{\text {l }}$ are non-zero, whereas for $1.6<J_{1}<1.82$, the gs transitions to a magnetic state. For $J_{1} \geq 1.82$, the gs spin equals twice the number of unit cells in the system. (b) $S_{G}$ is shown as a function of system sizes for $J_{1}=1.0$ and 2.0. Each data point corresponds to integer number of unit cells. (c) $S_{G}$ vs $J_{1}$ for different system sizes.

The linear variation for $S_{G}$ vs $N$ for $J_{1}=2$ is shown in Fig. 2(b). The finite size effect of $S_{G}$ is shown in $S_{G}$ vs $J_{1}$ curve for three system sizes $N=14,26$, and 50 in Fig. 2(c). We notice that $S_{G}$ increases in a stepwise fashion for a small system size but shows a sharp increase as the system size increases.

In small $J_{1}$ limit, spin correlation $C(r)$ behavior is similar to that of a HAF spin-1 chain with a short correlation length. In Figs. 3(a) and 3(b), the total correlation function $C(R, R+2 r)$ is shown for $J_{1}=1.0$ and 2.0, respectively. There are two unique sites one at the base of the triangle and other at the apex of the triangle. We consider reference sites on the middle triangle of the ladder with site labels 49,50 , and 51 of a $N=98$ site system. Sites 49 and 51 are at the base of the triangle, whereas site 50 is at the apex of the triangle, and correlations from these points $C(49,49+2 r)$ and $C(51,51+2 r)$ are the correlations between the reference sites on the lower leg of the ladder with all the sites on the lower leg, whereas $C(50,50+2 r)$ are the correlations between the reference site on the upper leg of the ladder with all the other sites on the upper leg. $C(R, R+2 r)$ for $R=49$ and 51 sites are similar as both these sites belong to the base of the triangle and are equivalent by symmetry. In Fig. 3(a), $C(R, R+2 r)$ for $J_{1}=1.0$ seems to show oscillatory behavior with exponentially decreasing amplitudes. 


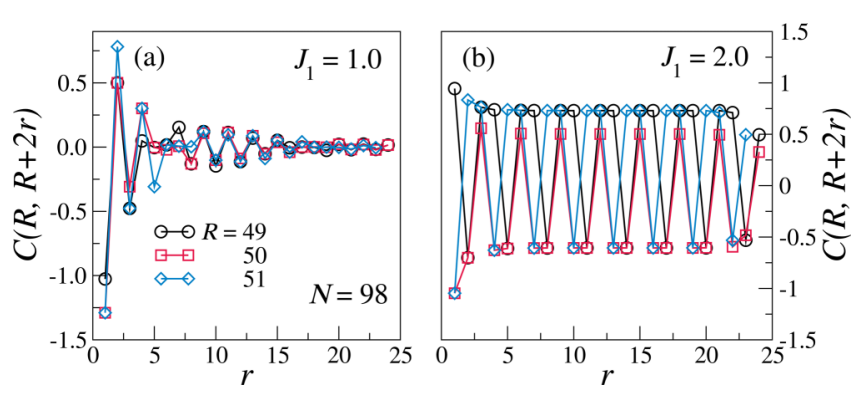

FIG. 3. The spin-spin correlations between the spins in the lower leg $(R=49$ and $R=51$ ), spins in upper leg $(R=50)$ for a $3 / 4$ ladder with $N=98$ spins with $\mathrm{OBC}$ with (a) $J_{1}=1.0$, the singlet regime, and (b) $J_{1}=2.0$, the magnetic regime.

This seems to indicate the existence of spin wave packets in the singlet state. $C(R, R+2 r)$ in the magnetic regime $J_{1}=2.0$ is shown Fig. $3(\mathrm{~b})$, and spins are antiferromagnetically aligned and have long range correlations. In this regime, in the bulk of the system, the correlations are oscillating with almost constant amplitude. The correlation between the reference spins in the base of the triangle is always ferromagnetic with spins at the base of the triangle and antiferromagnetic with the spin at the apex of the triangle. This is true on both legs. We have also computed the spin densities in the gs of the ladder as a function of $J_{1}$. The spin densities vanish at all the sites in the singlet gs. We note from Fig. 4 that the apex and the base sites have opposite spin densities in the magnetic gs. The spin densities at all the sites are nearly constant. Indeed, the spin-spin correlations in the magnetic state are well approximated by the product of the spin densities at the corresponding sites.

In the large $J_{1}$ limit, the spin densities show that the gs behavior can be thought of as each triangle having effective spin-1, which are interacting ferromagnetically. The spin density of an isolated triangle with large $J_{1}$ interaction is also 0.75 on the basal sites and -0.5 at the apex, which is close to the values found in the gs at large $J_{1}$.

In the $3 / 4$ system, there are only three distinct bond orders corresponding to the rung bond $b_{r}$, the bond between the basal sites $b_{b}$ and the bond between the apical and base sites $b_{a}$. We notice that both types of bonds on the legs $\left(b_{a}\right.$ and $\left.b_{b}\right)$ show decreasing bond order as $J_{1}$ increases and the basal bond order shows jump at $J_{1}=1.65$, whereas the magnitude of rung bond order increases continuously with $J_{1}$ and jumps suddenly at $J_{1}=1.65$. The jump in the rung bond order is due to the transition of the gs from a singlet to a magnetic state. The saturation value of the three bonds for the large $J_{1}$ limit is $+1.5,+1.0$ and -1.0 , respectively. The value $b_{r}=+1.5$ corresponds to two spin- $1 / 2$ objects separately forming bonds and total bond order is twice that of a singlet bond order $+3 / 4 . b_{b}=+1$ and $b_{a}=-1$ correspond to the ferromagnetic bond formation between axial and basal sites and intermediate spin state between the basal sites. In small $J_{1}$ limit, the $b_{r}$ rung bond is weak and $b_{r}$ is close to zero. Whereas $b_{b}$ and $b_{a}$ for small values of $J_{1}$ start with a value close to the bond order of a spin- 1 chain $\sim 1.40$, they decrease with increasing $J_{1} ; b_{b}$ becomes ferromagnetic and $b_{a}$ remains antiferromagnetic. (a)
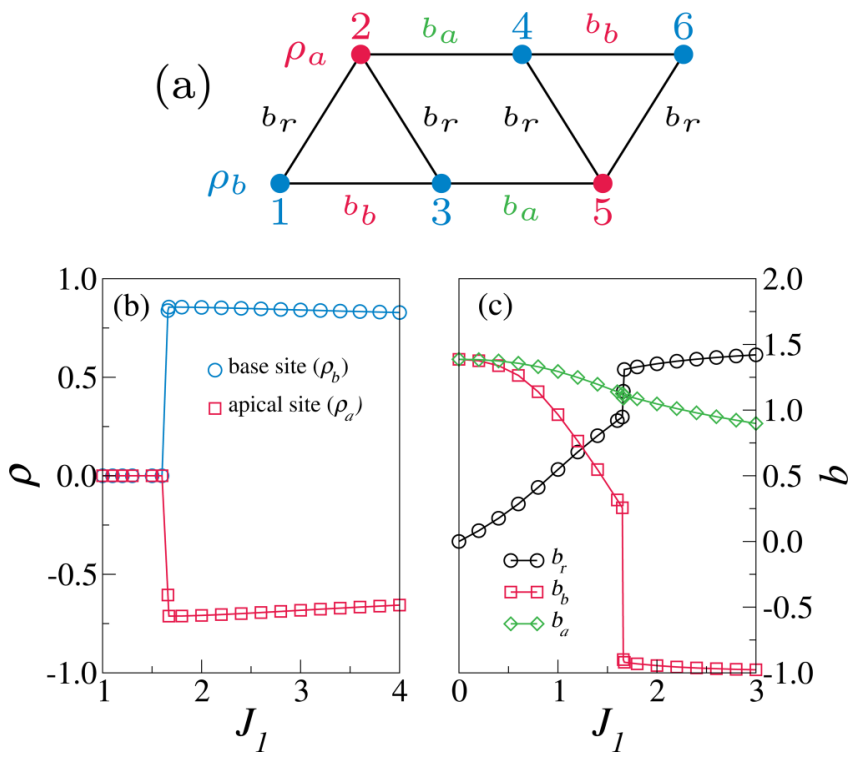

FIG. 4. (a) The base and the apical spins are shown in blue and red; three different types of bonds, namely, the rung bonds $\left(b_{r}\right)$, the basal bonds $\left(b_{b}\right)$, and the apical bonds $\left(b_{a}\right)$, are shown for a unit cell (b) Spin densities $\rho_{b}$ and $\rho_{a}$ for the base sites and the apical sites, respectively, as a function of $J_{1}$ (c) bond orders $b_{r}, b_{b}$, and $b_{a}$ as a function of $J_{1}$ are shown.

To understand the spin density and bond order behavior in large $J_{1}$ limit, we analyze a system with three spins on a triangle, and the exchange interactions of the two sides and base are $J_{1}$ and $J_{2}=1$, respectively. The gs is in spin- 1 states and there are six possible spin configurations. For $J_{1} \geq 2$, we calculate the gs wavefunction of the system in the $S=1$ and $S^{z}=1$ manifold as

$$
\begin{aligned}
\left|\Psi\left(S=1, S^{z}=1\right)\right\rangle= & \sqrt{\frac{3}{5}}\left[|1,-1,1\rangle+\frac{1}{2}(|0,0,1\rangle+|1,0,0\rangle)\right. \\
& \left.-\frac{1}{6}(|-1,1,1\rangle+|1,1,-1\rangle)-\frac{1}{3}|0,1,0\rangle\right]
\end{aligned}
$$

We notice that the large contribution $(60 \%)$ is from the state $|1,-1,1\rangle$ and a smaller (15\%) contribution is from the linear combination $1 / 2(|0,0,1\rangle+|1,0,0\rangle)$ spin configuration. Sites 1 and 3 are symmetric, and the total contribution of spin density comes from these two configurations and $60 \%$ contribution to $\left\langle S^{z}\right\rangle$ arises from $|1,-1,1\rangle$ while a contribution of $15 \%$ to $\left\langle S^{z}\right\rangle$ arises from $(|0,0,1\rangle+|1,0,0\rangle)$, resulting in a total spin density of 0.75 . The configuration $|1,-1,1\rangle$ contributes -0.6 to $\left\langle S^{z}\right\rangle$ at site 2 while the states $(|-1,1,1\rangle+|1,1,-1\rangle)$ and $|1,-1,1\rangle$ contribute +0.1 to $\left\langle S^{z}\right\rangle$ at site 2 . This results in total spin density at site 1 and 2 of 0.75 and -0.5 , respectively, in the large $J_{1}\left(J_{1} \geq 2.0\right)$ limit. The contribution to the $z$ component of all three bond orders mostly arises from the configuration $|1,-1,1\rangle$ and the bonds 1-2 and 2-3 are singlet in nature, whereas the bond $1-3$ is ferromagnetic. 


\section{B. 3/5 skewed ladder}

The 3/5 ladder has four spins per unit cell as shown schematically in Fig. 1(c), and in each unit cell, three sites form a triangle, whereas the fourth site is connected to the apex of the triangle. There is one rung bond at each odd-numbered site and two rung bonds at alternate even numbered sites, $(4 k-2), k=1,2, \ldots$. With periodic boundary condition, the sites $2 k$ at the apices of triangles and pentagons are inversion centers. The model Hamiltonian of this system is shown in Eq. (2)

The gs of the $3 / 5$ system is singlet in low $J_{1}$ limit and toggles between singlet and different magnetic states as $J_{1}$ is increased. We find that the evolution of the spin of the gs depends not only on $J_{1}$ but also on the system size. We show this dependence for four representative sizes of the systems with open boundary condition. For the system with six unit cells (Fig. 5) $(N=26)$, the gs switches from a singlet to a triplet at $J_{1}=0.84$ and it then switches back to a singlet at $J_{1}=1.7$. The triplet then becomes a gs from $J_{1}=2.15$ to $J_{1}=3.0$. Beyond $J_{1}=3.0$, the gs is a quintet until $J_{1}=6.5$, a septet $(S=3)$ from $J_{1}=6.5$ to 7.0 , a nonet $(S=4)$ from $J_{1}=7.0$ to 8.5 and $S=5$ beyond $J_{1}=8.5$. The highest spin in the $N=26$ systems with six unit cells can be $S=6$, corresponding to the ferromagnetic arrangement of spins at sites $4 k$. In large systems, these transitions occur at different values of $J_{1}$ (Fig. 5).

To understand this seemingly strange behavior, we have computed spin densities and spin-spin correlations for the gs with different spins (corresponding to different $J_{1}$ values) for the $N=98$ system. We see from Fig. 6 that in the interior of the system, higher spin densities are found at $4 k$ sites. In addition, the spin densities of a given type of sites show a wavelike behavior. Thus, the gs spin is dictated by two factors. Large $J_{1}$ favors a high spin gs, while the wavy nature of the spin density favors a standing wave as interference effects will be lowest in this state and so will be the spin fluctuations, which tend to increase the energy of a state. Hence, the gs shows the unusual spin state changes as $J_{1}$ is increased. This also explains the nonmonotonic dependence on the switching values of $J_{1}$ for different system sizes. When $J_{1}$ is very large $\left(J_{2} / J_{1} \rightarrow 0\right)$, we expect the gs spin of the system to be $4 n$, where $n$ is the number of unit cells in the system. This unusual behavior is also reflected in the spin-spin correlation function (Fig. 7), where the wavelength of
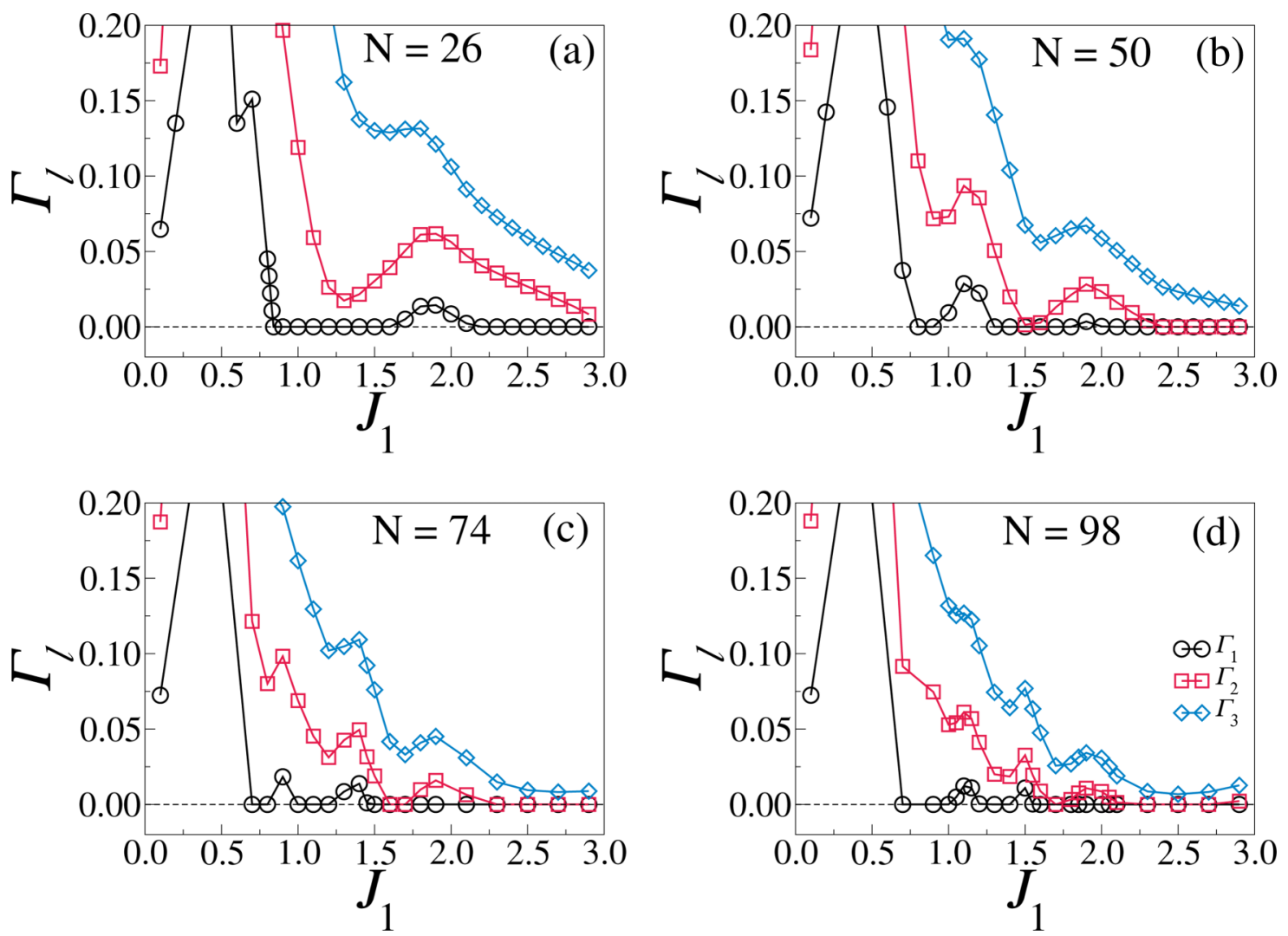

FIG. 5. The lowest excitation energy gaps in different $S^{z}$ sectors, $\Gamma_{l}$ for different $I=S^{z}$ manifolds as a function of the rung exchange interaction $J_{1}$ for systems of (a) $N=26$ spins ( 6 unit cells), (b) $N=50$ spins (12 unit cells), (c) $N=74$ spins (18 unit cells), and (c) $N=98$ (24 unit cells) with open boundary condition. For larger system sizes, the gs switches between magnetic and singlet states as $J_{1}$ is increased. 

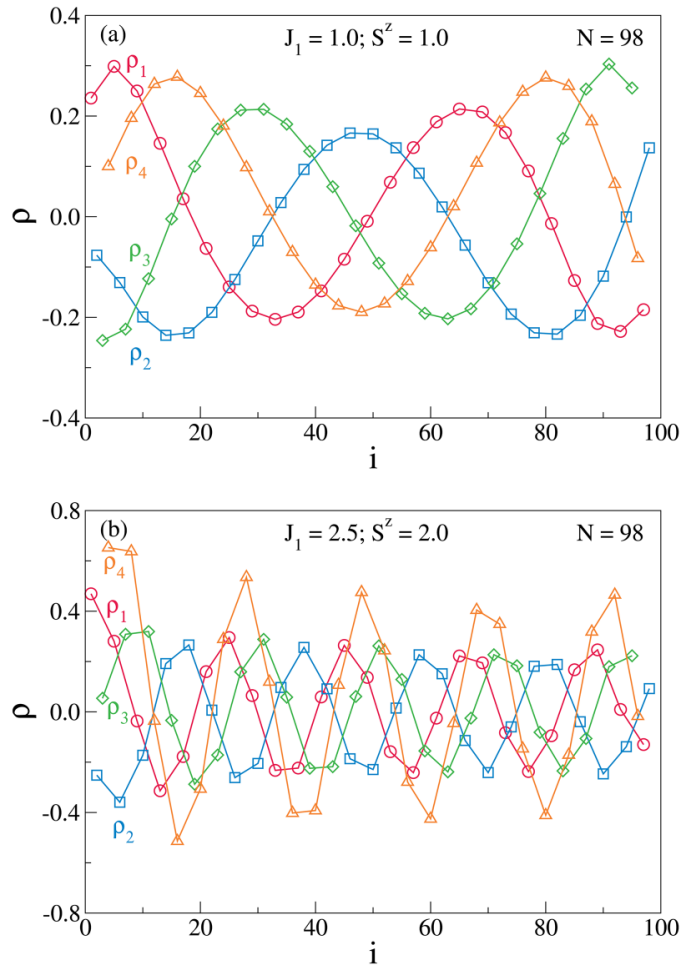

FIG. 6. Spin densities of a $3 / 5$ ladder of $N=98$ spins (OBC). (a) Spin densities for $J_{1}=1.0$ in the triplet gs are shown with continuous red, blue, green, and orange curve (the eye guide) for 1, 2, 3, and 4 sites in every unit cells. (b) Spin densities for $J_{1}=2.5$ are shown in the quintet gs. We have chosen $S^{z}=S_{G}$ in both cases. the correlation function decreases with $J_{1}$ and the magnitude of the correlation between spins at sites $4 k$ has a larger amplitude than the rest of the correlations.

In the case of our 3/5 ladder system, there are two mirror planes, one which is passing through the center of the five membered ring along the upper leg and perpendicular to the lower leg, whereas the second mirror plane " $\sigma$ " passes through the apex of the triangle and bisects the base triangle. The system also has spin inversion symmetry $P$, which exists in the $S^{z}=0$ subspace and corresponds to the invariance of the Hamiltonian when all the spins in the system are rotated by $\pi$ around the $y$ axis. This is equivalent to the invariance of Hamiltonian under spin inversion. This symmetry divides the $S^{z}=0$ subspace into an even ("+") and an odd ("-") subspace with the even (odd) subspace spanning states with even (odd) spins. Thus, the even subspace consists of basis functions with even integer total spin $(S)$ and the odd subspace consists of basis functions with odd integer total spin $(S)$. The symmetry group of the $3 / 5$ skewed ladder consists of $E, P, \sigma$, and $\sigma P$ and all these elements commute with each other and form an abelian group. The irreducible representations of this group are denoted by $A^{+}, A^{-}, B^{+}$, and $B^{-} . A(B)$ corresponds to even (odd) space under $\sigma$ and $+(-)$ corresponds to even (odd) space under $P$. The spin inversion symmetry is broken when the lowest energy states with odd and even total spins are degenerate and reflection symmetry is broken when lowest energy levels in both $A$ and $B$ spaces are degenerate. Both the symmetries are broken when the lowest energy states in $A^{+}\left(A^{-}\right)$and $B^{-}\left(B^{+}\right)$spaces are degenerate. We show the degeneracy of singlet and triplet states at $J_{1}=0.8075$ and triplet and quintet at $J_{1}=1.2183$ in Table I. For these values of $J_{1}$, the reflection symmetry is also broken as lowest energy states in spaces odd and even under reflections are also degenerate for these $J_{1}$ values. Hence, we observe spontaneous spin current. The
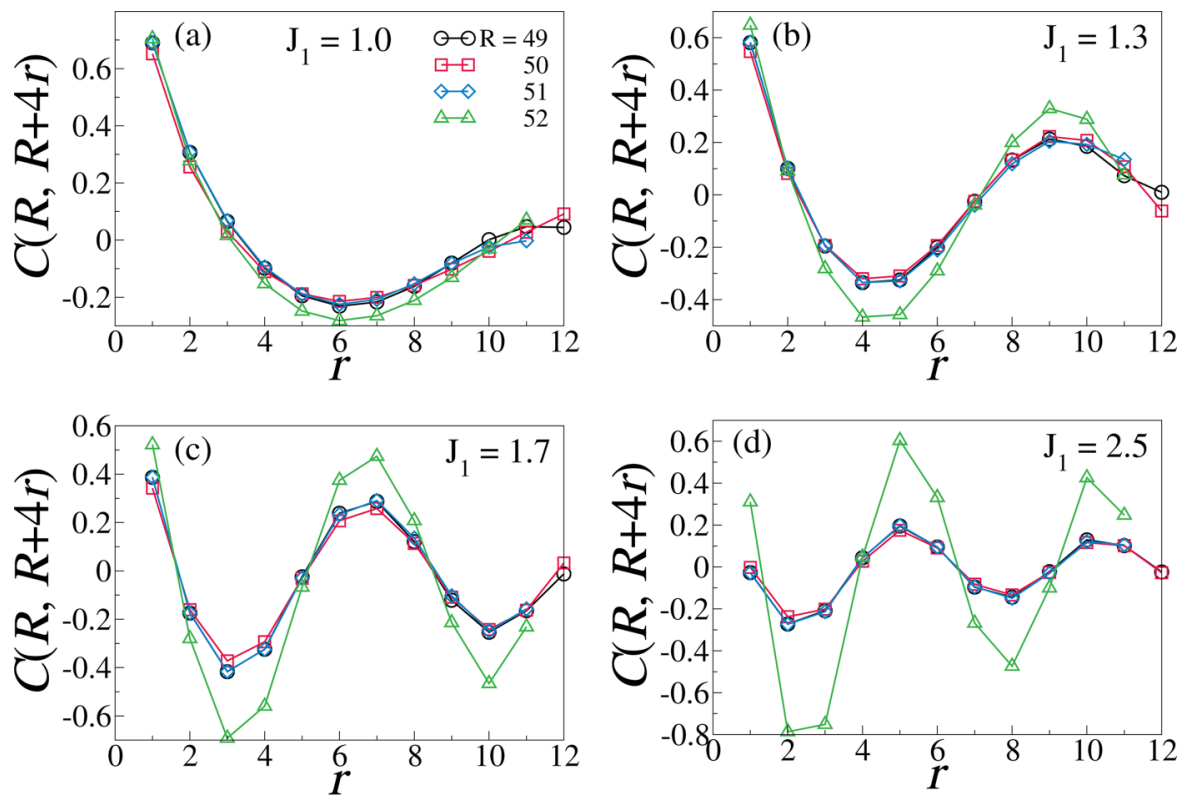

FIG. 7. Spin-spin correlations between spins at same type of sites in each unit cell of a $3 / 5$ ladder of $N=98$ spins (OBC) for (a) $J_{1}=1.0$, (b) $J_{1}=1.3$, (c) $J_{1}=1.7$, and (d) $J_{1}=2.5$. Starting from the middle of the ladder $R=49,50,51,52$ are the reference sites. 
TABLE I. Lowest energy levels for different $S_{z}$ values of $N=16$.

\begin{tabular}{llll}
\hline \hline$J_{1}$ & $E\left(S^{z}=0\right)$ & $E\left(S^{z}=1\right)$ & $E\left(S^{z}=2\right)$ \\
\hline 0.8705 & -23.0172 & -23.0172 & \\
& -23.0172 & & \\
1.2183 & -23.7554 & -23.7554 & -23.7554 \\
& -23.7554 & -23.7554 & \\
\hline \hline
\end{tabular}

$z$-component of the spin current is defined as

$$
\begin{aligned}
\kappa^{z}(j, k) & =-i\left\langle\Psi_{G}(-)\left|\left(\vec{S}_{j} \times \vec{S}_{k}\right)^{z}\right| \Psi_{G}(+)\right\rangle \\
& =\frac{1}{2}\left\langle\Psi_{G}(-)\left|\left(S_{j}^{+} S_{k}^{-}-S_{j}^{-} S_{k}^{+}\right)\right| \Psi_{G}(+)\right\rangle,
\end{aligned}
$$

which is the eigenvalue of the spin current operator $\left(\vec{S}_{j} \times \vec{S}_{k}\right)^{z}$ expressed as a matrix in the degenerate $\left|\Psi_{G}(+)\right\rangle$ and $\left|\Psi_{G}(-)\right\rangle$ basis where the function $\left|\Psi_{G}(+)\right\rangle\left(\left|\Psi_{G}(-)\right\rangle\right)$ is the gs in the even (odd) subspace for reflection and even (odd) subspace for spin inversion. In Fig. 8, we show the spin currents for $J_{1}=0.8705$ and 1.2183. The current in the triangle is counterclockwise, whereas it is clockwise in the pentagons and weak in the upper leg of pentagons. Interestingly, the spin current retains the qualitative features for both the $J_{1}$ values.

\section{SUMMARY AND CONCLUSIONS}

In this paper, we study the gs properties of spin-1 Heisenberg antiferromagnetic model on $3 / 4$ and $3 / 5$ skewed ladder geometries shown in Figs. 1(b) and 1(c). The 3/4 spin ladder system goes from a singlet state to a partially magnetized state. For $J_{1} \geq 1.82$ the magnetization per unit cell is $\langle m\rangle=2$ with each triangle in the system contributing $\langle m\rangle=1$ to the magnetization in the gs. The gs of this system for $J_{1}<1.6$ is a singlet and shows short range correlations. In this system, there are two unique sites and three unique

(a)

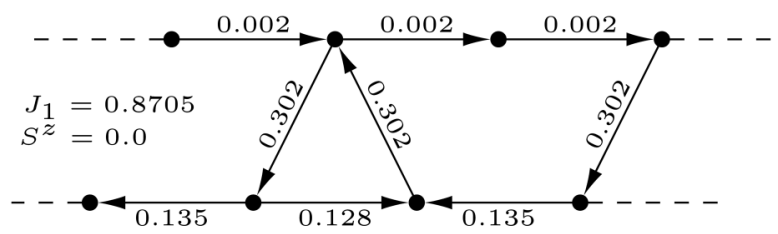

(b)

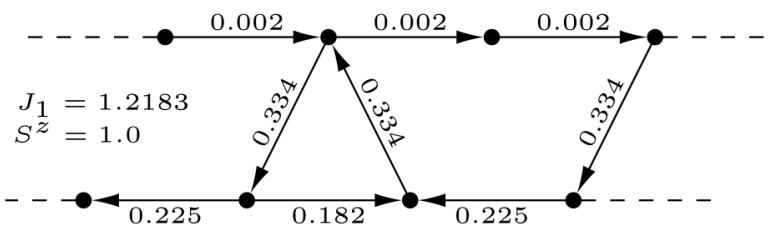

FIG. 8. The spin current in a $3 / 5$ skewed ladder of $N=16$ spins with periodic boundary condition at (a) $J_{1}=0.8705$ and (b) $J_{1}=1.2183$. The direction of the spin current is indicated by arrowheads and the magnitude is given by the numbers adjacent to the arrows. bonds in the six site unit cell. In the weak $J_{1}$ limit, the gs is a singlet and spin densities are zero and bond orders along the leg are close to that of the valance bond state of a spin-1 chain. The bond order in the upper leg that connects the apex or base of two different triangles decreases gradually as $J_{1}$ is increased while the bond order for the base of the triangle increases rapidly. In large $J_{1}$ limit or in magnetic gs, the spin densities for base and apex are 0.75 and -0.5 , respectively, and the effective spin per triangle is 1 . The bond order along the rung and the base of the triangle is 1.5 or equivalent to two spin-1/2 dimers and -1 for the bond between a basal site and an apical site, indicating a ferromagnetic interaction. In the large $J_{1}$ limit, the effective spin on each triangle interacts ferromagnetically. In the spin-1/2 skewed ladder system, the ferromagnetic arrangement of spin is an example of the McConnell mechanism ${ }^{45,52}$ with antiferromagnetic exchange (here $J_{2}$ ) between sites with positive and negative spin densities leading to a ferromagnetic interaction between the unpaired spins. The effective interaction between the spins in two neighboring triangles can be written as $J_{\text {eff }}=2 J_{2} \rho_{a} \rho_{b}$, where $\rho_{a}$ and $\rho_{b}$ are the spin densities at $a$ and $b$ type sites. We note that the ferromagnetic mechanism for the spin-1 system seems similar to the spin-1/2 system.

The gs properties of the $3 / 5$ ladder seem more complicated unlike the $3 / 4$ system. The gs is a singlet for $J_{1}<0.84$ and shows the spin density wave gs for larger $J_{1}$ values. What is interesting is that the gs spin $S_{G}$ varies between 0,1 , and 2 before attaining the saturation value of $n$ (the number of unit cells) for very large $J_{1}$. The switching of the gs depends on the system size and there is no apparent systematics. Analysis of spin densities and spin-spin correlations seem to indicate that there is a competition between the standing spin density wave of a lower spin state and the higher exchange stability of the successively higher spin state. This seems to render a qualitative explanation of the seemingly unsystematic switching in the gs spin of the system as a function of system size. This system also shows vector chiral phase due to simultaneous breaking of the reflection symmetry and spin inversion symmetry. The broken symmetry phase is characterized by nonzero spin currents in the system.

In summary, we have studied the gs properties of an antiferromagnetic Heisenberg spin-1 system on $3 / 4$ and $3 / 5$ skewed ladders. Both systems transition from singlet to a partially polarized gs on tuning $J_{1}$. The $3 / 5$ system shows switching of the spin state between singlet and different magnetic states due to a competition between $J_{1}$, which favors a high spin state, and standing spin density wave, which favors a lower spin state. For two different parameter values, the gs of the system has doubly degenerate gs, which leads to the breaking of spin-parity and reflection symmetry in the system resulting in spontaneous spin current. Although such systems have not yet been experimentally realized, we believe that they can be realized in molecular magnets based on transition metal compounds.

\section{AUTHORS' CONTRIBUTIONS}

S.D. and D.D. contributed equally to this work. 


\section{ACKNOWLEDGMENTS}

M.K. acknowledges the SERB for financial support through Project File No. CRG/2020/000754. S.R. acknowledges the Indian National Science Academy and DST-SERB for supporting this work.

\section{DATA AVAILABILITY}

The data that support the findings of this study are available from the corresponding author upon reasonable request.

\section{REFERENCES}

'F. D. M. Haldane, Phys. Lett. 93A, 464 (1983).

${ }^{2}$ F. D. M. Haldane, Phys. Rev. Lett. 50, 1153 (1983).

${ }^{3}$ I. Affleck, T. Kennedy, E. H. Lieb, and H. Tasaki, Phys. Rev. Lett. 59, 799 (1987).

${ }^{4}$ I. Affleck, T. Kennedy, E. H. Lieb, and H. Tasaki, Commun. Math. Phys. 115, 477 (1988).

${ }^{5}$ C. K. Majumdar and D. K. Ghosh, J. Math. Phys. 10, 1388 (1969).

${ }^{6}$ C. K. Majumdar and D. K. Ghosh, J. Math. Phys. 10, 1399 (1969).

${ }^{7}$ S. R. White and I. Affleck, Phys. Rev. B 54, 9862 (1996).

${ }^{8}$ M. Kumar, A. Parvej, and Z. G. Soos, J. Phys.: Condens. Matter 27, 316001 (2015).

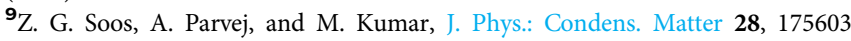
(2016).

${ }^{10}$ T. Hamada, J.-I. Kane, S.-I. Nakagawa, and Y. Natsume, J. Phys. Soc. Jpn. 57, 1891 (1988)

${ }^{11}$ A. V. Chubukov, Phys. Rev. B 44, 4693 (1991).

${ }^{12}$ R. Chitra, S. Pati, H. R. Krishnamurthy, D. Sen, and S. Ramasesha, Phys. Rev. B 52, 6581 (1995).

${ }^{13}$ C. Itoi and S. Qin, Phys. Rev. B 63, 224423 (2001).

${ }^{14}$ F. Heidrich-Meisner, A. Honecker, and T. Vekua, Phys. Rev. B 74, 020403(R) (2006).

${ }^{15}$ F. Heidrich-Meisner, I. A. Sergienko, A. E. Feiguin, and E. R. Dagotto, Phys. Rev. B 75, 064413 (2007).

${ }^{16}$ L. Kecke, T. Momoi, and A. Furusaki, Phys. Rev. B 76, 060407(R) (2007).

${ }^{17}$ T. Vekua, A. Honecker, H.-J. Mikeska, and F. Heidrich-Meisner, Phys. Rev. B 76, 174420 (2007).

${ }^{18}$ S. Mahdavifar, J. Phys.: Condens. Matter 20, 335230 (2008).

${ }^{19}$ D. V. Dmitriev and V. Y. Krivnov, Phys. Rev. B 77, 024401 (2008).

${ }^{20}$ T. Hikihara, L. Kecke, T. Momoi, and A. Furusaki, Phys. Rev. B 78, 144404 (2008).

${ }^{21}$ J. Sudan, A. Lüscher, and A. M. Läuchli, Phys. Rev. B 80, 140402(R) (2009).
${ }^{22}$ F. Heidrich-Meisner, I. P. McCulloch, and A. K. Kolezhuk, Phys. Rev. B 80, 144417 (2009).

${ }^{23}$ J. Sirker, Phys. Rev. B 81, 014419 (2010).

${ }^{24}$ M. Kumar, S. Ramasesha, and Z. G. Soos, Phys. Rev. B 81, 054413 (2010).

${ }^{25}$ M. Kumar and Z. G. Soos, Phys. Rev. B 85, 144415 (2012).

${ }^{\mathbf{2 6}}$ A. Parvej and M. Kumar, Phys. Rev. B 96, 054413 (2017).

${ }^{27}$ H.-J. Mikeska and A. K. Kolezhuk, "One-dimensional magnetism," in Quantum Magnetism, edited by U. Schollwöck, J. Richter, D. J. J. Farnell, and R. F. Bishop (Springer, Berlin, 2004), pp. 1-83.

${ }^{28}$ U. Schollwöck, O. Golinelli, and T. Jolicœur, Phys. Rev. B 54, 4038 (1996).

${ }^{29} \mathrm{~S}$. Östlund and S. Rommer, Phys. Rev. Lett. 75, 3537 (1995).

${ }^{30}$ F. Verstraete, V. Murg, and J. Cirac, Adv. Phys. 57, 143 (2008).

${ }^{31}$ U. Schollwöck, Ann. Phys. 326, 96 (2011).

${ }^{32}$ R. Orús, Ann. Phys. 349, 117 (2014).

${ }^{33}$ V. Murg, F. Verstraete, and J. I. Cirac, Phys. Rev. A 75, 033605 (2007).

${ }^{34}$ F. Verstraete and J. I. Cirac, Phys. Rev. A 70, 060302(R) (2004).

${ }^{35}$ T.-C. Wei, I. Affleck, and R. Raussendorf, Phys. Rev. Lett. 106, 070501 (2011).

${ }^{36}$ J. P. Renard, M. Verdaguer, L. P. Regnault, W. A. C. Erkelens, J. Rossat-Mignod, and W. G. Stirling, Europhys. Lett. 3, 945 (1987).

37J. P. Renard, M. Verdaguer, L. P. Regnault, W. A. C. Erkelens, J. Rossat-Mignod, J. Ribas, W. G. Stirling, and C. Vettier, J. Appl. Phys. 63, 3538 (1988).

${ }^{38}$ K. Katsumata, H. Hori, T. Takeuchi, M. Date, A. Yamagishi, and J. P. Renard, Phys. Rev. Lett. 63, 86 (1989).

${ }^{39}$ D. Dey, M. Kumar, and Z. G. Soos, Phys. Rev. B 94, 144417 (2016).

${ }^{40} \mathrm{~S}$. R. White and D. A. Huse, Phys. Rev. B 48, 3844 (1993).

${ }^{41}$ S. Todo, M. Matsumoto, C. Yasuda, and H. Takayama, Phys. Rev. B 64, 224412 (2001).

${ }^{42}$ T. Hikihara, M. Kaburagi, and H. Kawamura, Prog. Theor. Phys. Suppl. 145, 58 (2002).

${ }^{43}$ N. Chepiga, I. Affleck, and F. Mila, Phys. Rev. B 93, 241108(R) (2016).

${ }^{44}$ S. Thomas, S. Ramasesha, K. Hallberg, and D. Garcia, Phys. Rev. B 86, 180403 (R) (2012)

${ }^{45}$ G. Giri, D. Dey, M. Kumar, S. Ramasesha, and Z. G. Soos, Phys. Rev. B 95, 224408 (2017).

${ }^{46}$ D. Dey, S. Das, M. Kumar, and S. Ramasesha, Phys. Rev. B 101, 195110 (2020).

${ }^{47}$ S. Das, D. Dey, M. Kumar, and S. Ramasesha, "Quantum phases of a frustrated spin-1 system: The 5/7 skewed ladder," arXiv:2012.07486 [cond-mat.str-el] (2020).

${ }^{48}$ S. R. White, Phys. Rev. Lett. 69, 2863 (1992)

${ }^{49}$ S. R. White, Phys. Rev. B 48, 10345 (1993).

${ }^{50}$ U. Schollwöck, Rev. Mod. Phys. 77, 259 (2005).

${ }^{\mathbf{5 1}}$ K. A. Hallberg, Adv. Phys. 55, 477 (2006).

${ }^{52}$ H. M. McConnell, J. Chem. Phys. 39, 1910 (1963). 